\title{
Locked Nucleic Acid (LNA)-based Nucleic Acid Sensors
}

\section{Sourav Mishra and Rupa Mukhopadhyay*}

Department of Biological Chemistry, Indian Association for the Cultivation of Science, Jadavpur, Kolkata-700 032, India

In recent times, much developments in the field of 'nucleic acid sensors', especially using alternative nucleic acid probes like peptide nucleic acid (PNA) and locked nucleic acid (LNA), have taken place. Although most of these are concerned with 'solution phase' studies, some reports have been made on 'on-surface' detection. The latter type of detection is particularly important to nurture, considering clinical diagnostic applications using microarrays. In this article, we'll briefly present the primary developments reported in the past two decades, along with possibilities for future developments, in case of the LNAbased sensors.

Among the different definitions of biosensors available so far, the most accepted and updated version (by IUPAC) is "a biosensor is a compact analytical device incorporating a biological or biologically derived sensing element, either integrated within or intimately associated with a physicochemical transducer" [1]. Nucleic acid based biosensors are those in which the probe molecule is DNA, RNA, or synthetic polymer analogous to natural nucleic acids like PNA, LNA etc. [2]. Most of the current nucleic acid based biosensors exploit their base pair hybridization properties, although some use aptamers as biosensing element [3]. Nucleic acid immobilization on the biosensor surface is a crucial step that affects the overall performance of the sensor. In general nucleic acids are immobilized onto solid surfaces in such a way that a signal is obtained only if they react with their specific target molecules. The immobilization methods include covalent binding (immobilization onto the surface via one end of the nucleic acid molecule, e.g., binding of a $5^{\prime}$-amino-modified DNA oligonucleotide onto an epoxy-modified surface), non-covalent binding (e.g., affinity binding as relevant in case of avidin-biotin interactions), and chemisorption via near-covalent interactions (e.g., adsorption of thiolated oligonucleotides on gold surface via formation of goldsulfur linkages) [2]. For signal transduction in the current nucleic acid sensors, without compromising with sensitivity and specificity of target detection, optimized electrochemical, electrical, optical, mechanical, acoustic, or thermal methods are applied $[4,5]$.

In the past two decades, the nucleic acid based biosensor technologies have made considerable advancements and have been applied in important areas like gene expression profiling, genotyping, and biological detection. Major investigations so far mainly focused on DNA-based sensors. Though the DNA-based sensors have found wide applications in the microscale [6] as well as nanoscale nucleic acid sensing experiments [7-10], its reduced bioactivity due to non-specific DNA-surface interactions through relatively exposed nucleobases and degradability by the nuclease compel one to search for alternatives. Locked Nucleic Acid (LNA) is a conformationally restricted molecule since it contains a modified ribose moiety in which the $2^{\prime}$-oxygen and the $4^{\prime}$-carbon are linked by a methylene bridge - in effect, locking the sugar in a RNA mimicking sugar conformation (N-type) [11,12]. LNA can bind with complementary DNA/RNA sequences in a sequencespecific manner obeying the Watson-Crick base pairing rule with higher affinity compared to DNA, which is reflected in the higher values of the solution melting temperatures of LNA-DNA/LNA-RNA duplexes compared to those of DNA-DNA/DNA-RNA duplexes $[11,12]$. LNA is nuclease-resistant; $[13,14]$ its higher structural rigidity may prevent interactions with the solid substrates, [15] and it can have multiple water bridges that provide it with extra stability compared to DNA or RNA. It has been shown that both the highest $T_{m}$ increase per LNA modification and the best mismatch discrimination are achieved for short LNA sequences [16]. In addition to that, LNA phosphoramidites and their oligomers are commercially available, and LNA nucleotides can be mixed with those of the natural nucleic acids for generating heterogeneous probe molecules. These properties of LNA hold the promise that it can be a potentially better alternative than DNA in nucleic acid based detection technologies.

In early days, Orum et al. demonstrated that LNAs can effectively and reproducibly capture PCR amplicons in a simple solidphase hybridization assay [17]. Fang et al. [18] reported that LNA microarrays that could be used to detect multiple miRNAs by means of a novel approach have an outstanding detection limit of approximately $10^{-18} \mathrm{~mol} \mathrm{~L}^{-1}$. Simeonov and Nikiforov [19] reported efficient SNP scoring using short LNAs by means of fluorescence polarization (FP) detection. Wang et al. [20] designed molecular beacons (MB) with a LNA backbone to generate novel probes with higher thermostability, enhanced selectivity, nuclease resistance, and reduction of false positive signals, even in complex biological environments. Chen et al. [21] used a 18-mer LNA-modified capture probe for hybridization with the BCR/ ABL fusion gene to detect chronic myelogenous leukaemia. Differential pulse voltammetry was used to monitor the hybridization reaction on the capture probe electrode. Recently, they dually labelled the LNA hairpin probe with biotin (for streptavidin-based immobilization) and a carboxyfluorescein (FAM) molecule (as an affinity tag for HRP) [22]. The immobilized hairpin probe suffers a significant conformational change upon target hybridization, separating FAM from the electrode and making it accessible to the anti-FAM-HRP antibody. This biosensor is capable of specific SNP detection and could be used to detect $8.3 \times 10^{-14} \mathrm{~mol} \mathrm{~L}^{-1}$ target DNA in real samples, thus constituting a good example of the usefulness of LNA-based probes. A comparative study of the properties of PNA and LNA as capture probes for development of an electrochemical hybridization assay has been carried out by Mascini's group [23]. Where, streptavidin-coated paramagnetic microbeads were used as solid phase to immobilize biotinylated DNA, PNA, and LNA capture probes complementary to DNA and RNA target oligonucleotides. Detection limits for the DNA target were 1.52, 1.18, and $0.91 \times 10^{-10} \mathrm{~mol} \mathrm{~L}^{-1}$ (DNA, PNA, and LNA probes, respectively). For the RNA target, they were even smaller: 5.1, 6.0, and $7.8 \times 10^{-11} \mathrm{~mol} \mathrm{~L}^{-1}$, respectively. While the performance of the biosensor for SNP detection

*Corresponding author: Rupa Mukhopadhyay, Department of Biological Chemistry Indian Association for the Cultivation of Science, Jadavpur, Kolkata-700 032, India, E-mail: bcrm@iacs.res.in

Received April 30, 2013; Accepted May 02, 2013; Published May 04, 2013

Citation: Mishra S, Mukhopadhyay R (2013) Locked Nucleic Acid (LNA)-based Nucleic Acid Sensors. J Bioanal Biomed 5: e114. doi:10.4172/1948-593X.1000e114

Copyright: @ 2013 Mishra S, et al. This is an open-access article distributed under the terms of the Creative Commons Attribution License, which permits unrestricted use, distribution, and reproduction in any medium, provided the original author and source are credited. 
was not checked in those cases, an application that is likely to reveal the advantages of PNA and LNA probes over DNA probes, was tested in such a biosensor. LNA nucleotides have also been introduced in DNA or RNA aptamers developed for biosensing applications, because "locked" nucleotides not only increase the thermal stability of the aptamer but also improve its in vivo resistance to nuclease digestion. Darfeuille et al. studied the effect of incorporation of LNA nucleotides into the RNA aptamer specific to the HIV-1 TAR RNA element [24].

Motivated by the earlier findings that LNA can potentially be a better alternative in nucleic acid detection technologies, and considering the lack of studies reported on on-surface nucleic acid detection by the LNA probes, the authors' group developed a sensitive and robust LNA sensor layer, which is capable of efficient and specific target detection. Systematic designing of such films requires molecular level understanding of the structural and functional properties of these films, since it is often found that the nature of the molecular organization can influence the film's capacity of being bioactive. We reported for the first time, a straightforward way (based on simple immersion method) of generating an ordered self-assembled LNA monolayer, which is bioactive, onto a gold (111) surface. This layer is capable of giving rise to a stronger DNA recognition signal (4-4.5 times) than its DNA counterpart, and importantly, it can differentiate between a fully complementary DNA target and that having a single base mismatch, where the mismatch discrimination ratio is almost two times compared to the ratio relevant in case of DNA-based detection [25]. Recently, we have further shown that by varying the salt concentration and type of cations, the single base mismatch discrimination ability of surface-tethered LNA probes could be controlled. At the same time, we proposed a method to measure the 'on-surface' melting temperature of nucleic acid duplexes formed in different ionic environments and then from the difference in melting temperatures the sensitivity of the approach was measured. Generally, it was observed that with increase in salt concentration mismatch discrimination ability of the LNA sensor layer was increased and $\mathrm{Mg}^{2+}$ was found to be more efficient in single base mismatch discrimination compared to the monovalent sodium as well as the trivalent spermidine and tetravalent spermine [26].

Though the studies mentioned above indicate that LNA probes are better performers than the DNA probes in some aspects, it's yet to be seen whether LNA-based microarrays can be developed for clinical purposes and especially if it can be realized for high-throughput detection. For this, highly reproducible, robust and sensitive detection is necessary and works in the authors' laboratory are in progress towards this direction.

\section{References}

1. Newman JD, Tigwell LJ, Turner APF, Warner PJ (2004) Biosensors: a clearer view. Cranfield University.

2. Labuda J, Oliveira Brett AM, Evtugyn G, Fojta M, Mascini M, et al. (2010) Electrochemical nucleic acid-based biosensors: concepts, terms, and methodology (IUPAC Technical report). Pure Appl Chem 82: 1161-1187.

3. Juskowiak B (2011) Nucleic acid-based fluorescent probes and their analytical potential. Anal Bioanal Chem 399: 3157-3176.

4. Rasooly A, Herold K (2009) Biosensors and biodetection: methods and protocols volume1: optical-based detectors. vol 503. Humana Press.

5. Rasooly A, Herold K (2009) Biosensors and biodetection: methods and protocols volume 2: electrochemical and mechanical detectors, lateral flow and ligands for biosensors vol 504. Humana Press.

6. Sassolas A, Leca-Bouvier BD, Blum LJ (2008) DNA biosensors and microarrays. Chem Rev 108: 109-139

7. Fritz J, Baller MK, Lang HP, Rothuizen H, Vettiger P, et al. (2000) Translating biomolecular recognition into nanomechanics. Science 288: 316-318.

8. Mukhopadhyay R, Lorentzen M, Kjems J, Besenbacher F (2005) Nanomechanical sensing of DNA sequences using piezoresistive cantilevers. Langmuir 21: 8400-8408.

9. Drummond TG, Hill MG, Barton JK (2003) Electrochemical DNA sensors. Nat Biotechnol 21: 1192-1199.

10. Hansen JA, Mukhopadhyay R, Hansen JÃ , Gothelf KV (2006) Femtomolar electrochemical detection of DNA targets using metal sulfide nanoparticles. J Am Chem Soc 128: 3860-3861.

11. Singh SK, Nielsen P, Koshkin AA, Wengel J (1998) LNA (locked nucleic acids) synthesis and high-affinity nucleic acid recognition. Chem Commun 455

12. Koshkin AA, Nielsen P, Singh SK, Wengel J (1998) LNA (Locked Nucleic Acid): An RNA Mimic Forming Exceedingly Stable LNA:LNA Duplexes. J Am Chem Soc 120: 13252 .

13. Vester B, Wengel J (2004) LNA (Locked Nucleic Acid): HighAffinity Targeting of Complementary RNA and DNA. Biochemistry 43: 13233

14. Kurreck J, Wyszko E, Gillen C, Erdmann VA (2002) Design of antisense oligonucleotides stabilized by locked nucleic acids. Nucleic Acids Res 30 1911-1918.

15. Martinez K, Estevez MC, Wu Y, Phillips JA, Medley CD, et al. (2009) Locked nucleic acid based beacons for surface interaction studies and biosensor development. Anal Chem 81: 3448-3454.

16. Petersen M, Wengel J (2003) LNA: a versatile tool for therapeutics and genomics. Trends Biotechnol 21: 74-81.

17. Orum H, Jakobsen MH, Koch T, Vuust J, Borre MB (1999) Detection of the factor $V$ Leiden mutation by direct allele-specific hybridization of PCR amplicons to photoimmobilized locked nucleic acids. Clin Chem 45: 1898-1905.

18. Fang S, Lee HJ, Wark AW, Corn RM (2006) Attomole microarray detection of microRNAs by nanoparticle-amplified SPR imaging measurements of surface polyadenylation reactions. J Am Chem Soc 128: 14044-14046.

19. Simeonov A, Nikiforov TT (2002) Single nucleotide polymorphism genotyping using short, fluorescently labeled locked nucleic acid (LNA) probes and fluorescence polarization detection. Nucleic Acids Res 30: e91.

20. Wang L, Yang CJ, Medley CD, Benner SA, Tan W (2005) Locked nucleic acid molecular beacons. J Am Chem Soc 127: 15664-15665.

21. Chen J, Zhang J, Wang K, Lin X, Huang L, et al. (2008) Electrochemica biosensor for detection of BCR/ABL fusion gene using locked nucleic acids on 4-aminobenzenesulfonic acid-modified glassy carbon electrode. Anal Chem 80: $8028-8034$

22. Lin L, Liu Q, Wang L, Liu A, Weng S, et al. (2011) Enzyme-amplified electrochemical biosensor for detection of PML-RARî fusion gene based on hairpin LNA probe. Biosens Bioelectron 28: 277-283.

23. Laschi S, Palchetti I, Marrazza G, Mascini M (2009) Enzyme-amplified electrochemical hybridization assay based on PNA, LNA and DNA probemodified micro-magnetic beads. Bioelectrochemistry 76: 214-220.

24. Darfeuille F, Hansen JB, Orum H, Di Primo C, Toulme JJ (2004) LNA/DNA chimeric oligomers mimic RNA aptamers targeted to the TAR RNA element of HIV-1. Nucleic Acids Res 32: 3101-3107.

25. Mishra S, Ghosh S, Mukhopadhyay R (2012) Ordered self-assembled locked nucleic acid (LNA) structures on gold(111) surface with enhanced single base mismatch recognition capability. Langmuir 28: 4325-4333.

26. Mishra S, Ghosh S, Mukhopadhyay R (2013) Maximizing mismatch discrimination by surface-tethered locked nucleic acid probes via ionic tuning Anal Chem 85: 1615-1623. 